\title{
Isolation and characterization of strong gene regulatory sequences from apple, Malus $\times$ domestica
}

\author{
Jan G. Schaart • Iris E. M. Tinnenbroek-Capel • \\ Frans A. Krens
}

Received: 8 January 2010 /Revised: 14 May 2010/Accepted: 12 July 2010 /Published online: 17 August 2010

(C) The Author(s) 2010. This article is published with open access at Springerlink.com

\begin{abstract}
For the strong expression of genes in plant tissue, the availability of specific gene regulatory sequences is desired. We cloned promoter and terminator sequences of an apple (Malus $x$ domestica) ribulose biphosphate carboxylase small subunit gene $(M d R b c S)$, which is known for its high expression and used gus reporter gene expression to test the regulatory activity of the isolated promoter and terminator sequences in transgenic tobacco. The $M d R b c S$ promoter itself seemed to be less strong than the CaMV35S promoter when both used in combination with the nos terminator. However, the combination of the promoter and terminator of $M d R b c S$ was able to drive gus to similar expression levels as the reference construct with CaMV35S promoter and nos terminator. This observation indicates the importance of the terminator sequence for gene expression. It is concluded that the combination of the $M d R b c S$ promoter and terminator is a suitable regulatory sequence set for the expression of transgenes to a high level in plants and for intragenesis in apple specifically.
\end{abstract}

Keywords Apple - Gene promoter and terminator. Genetic modification

\section{Introduction}

In the production of genetically modified (GM) crops that are to be directly consumed by humans, there is a tendency

Communicated by R. Velasco

J. G. Schaart $(\bowtie) \cdot$ I. E. M. Tinnenbroek-Capel · F. A. Krens

Wageningen UR Plant Breeding,

Wageningen University and Research Center,

P.O. Box 16, 6700AA Wageningen, The Netherlands

e-mail: jan.schaart@wur.nl to use preferentially genes and gene regulatory sequences that originate from plants themselves, rather than from, e.g., micro-organisms. Social studies aimed to elucidate factors determining consumer's attitude toward GM crops indicate that such crops are likely to be accepted much more readily. Lusk and Sullivan (2002) found that consumers would prefer GM vegetables with genes coming from the same species. Taking this concept as a starting point, different scenarios can be envisaged.

Important for the introduction of a new trait by genetic modification are the function of the gene that is used and the expression of that gene. Expression is regulated by genomic DNA sequences that flank the coding region at its 5 '- and 3 '-ends. The 5 ' upstream sequences or promoters determine the level of expression and the tissue specificity or timing, e.g., developmental stage or after induction by biotic or abiotic stress. For optimal desired performance, new combinations of coding regions and regulators might be made for genetic modification. Still, both types of DNA sequences can be taken from the recipient species. This type of genetic modification is referred to as intragenesis (Rommens et al. 2004). In case the gene or genes are used in their original configuration, which is with their own promoter and terminator regions, introns and in a sense orientation, this type of genetic modification has been called cisgenesis (Schouten et al. 2006; Schouten and Jacobsen 2008).

In our apple genetic modification program, a promoter was needed ensuring high level of transcription in many tissue types, comparable to the constitutive CaMV (cauliflower mosaic virus) $35 \mathrm{~S}$ promoter, but obtained from apple. The promoter of the gene coding for the small subunit of the ribulose biphosphate carboxylase (RBC) has been studied extensively (Khoudi et al. 1997; Nomura et al. 2000; Gittins et al. 2000; Outchkourov et al. 2003) in both 
homologous and heterologous crops and was found to be able to provide strong transcriptional activity. $\mathrm{RBC}$ is one of the most abundant proteins in the plant kingdom and can constitute $40-60 \%$ of total leaf protein. The enzyme is composed of eight small and eight large subunits encoded respectively by the nuclear and the chloroplast genome (Jensen and Bahr 1977). The RBC small subunit $(R b c S)$ genes constitute a gene family of 2-12 members that contribute to varying extents to the total transcriptional activity (Outchkourov et al. 2003). The expression of the genes is observed in photosynthetically active tissue and is regulated by light (Dean and Leech 1982). Expression levels of heterologous genes driven by $R b c S$ promoter sequences in different crops range between half that obtained by the CaMV35S promoter to seven to eightfold higher than CaMV35S (Outchkourov et al. 2003; Khoudi et al. 1997). In apple, Gittins et al. (2000) tested heterologous $R b c S$ promoters of tomato and soybean and concluded that they would be suitable for expression in green photosynthetic tissues. Also, the $R b c S$ promoter of chrysanthemum was tested in our laboratory in apple, and strong transcriptional activity could be demonstrated (F.A. Krens, pers. communication). However, these heterologous promoters are not suitable for intragenesis in apple.

Here, we describe the isolation and cloning of the $R b c S$ $5^{\prime}$ and $3^{\prime}$ regulatory sequences of apple, based on homology to $R b c S$ sequences of other crops, and the preparation of an expression cassette. The expression cassette was combined with the gus reporter gene and used in transformation of tobacco and apple. Expression levels were quantified in tobacco and were compared to the levels obtained using the constitutive CaMV35S promoter.

\section{Materials and methods}

\section{Isolation of genomic DNA}

Genomic DNA was isolated from young leaves of the apple cultivar Elstar according to the method described by Doyle and Doyle (1987), including 1\% (w/v) polyvinylpyrrolidone-10 in the DNA extraction buffer.

\section{Genome walking}

Isolation of $5^{\prime}$-end (promoter) and $3^{\prime}$-end (terminator) flanking genomic sequences of the apple $R b c S$ gene $(M d R b c S)$ was performed following a genome walking protocol. In short, eight different in vitro genomic DNA libraries of the apple cultivar Elstar were constructed by digesting genomic DNA with eight different blunt-end cutting restriction enzymes followed by a ligation of an adapter to the ends of the restriction fragments. The adapter is described by van der Linden et al. (2004). The libraries were used as templates in a genome-walking PCR in which, for the amplification of promoter fragments, two primers specific for the $5^{\prime}$-end of the $M d R b c S$ cDNA sequence (GenBank accession number L24497), pGSP1 (5'GTGAATGTGTGATGGTGGTTSGCCTCT-3') and pGSP2 (5'-AGGTTATCTATTAGGGTGCGTTTGTTG-3'), and for the amplification of terminator fragments, two primers specific for the 3 '-end of the $M d R b c S$ cDNA sequence, tGSP1 (5'-AGGGTCCCGGTGGTCTGTTTAAGTTTG-3') and tGSP2 (5'-TCGGATTCAAGATGGTGTTGGT TGAGA-3'), were used together with adapter-specific primers (van der Linden et al. 2004). Maximase ${ }^{\mathrm{TM}}$ Polymerase (Transgenomic) was used for amplification. Following a primary and nested PCR, different PCR fragments were obtained, and two putative promoter fragments of 0.9 and $1.6 \mathrm{~kb}$, amplified from an $H p a \mathrm{I}-$ and Hinc-digested genomic DNA library, respectively, and a putative terminator fragment of $1.1 \mathrm{~kb}$, amplified from a Hinc-digested genomic DNA library, were cloned in a PCR cloning vector pGEM-T Easy (Promega) and completely sequenced.

\section{Construction of transformation vectors}

For the construction of expression vectors, two $M d R b c S$ genomic fragments of 1,232 and 1,679 bp located directly upstream of the translational start and a single $M d R b c S$ genomic fragment of $582 \mathrm{bp}$ located directly downstream of the translational stop of $M d R b c S$ were amplified by PCR using genomic apple DNA as template (primers: 1.6-kb 5'end fragment: pMdRbcS1.6-FW, 5'-TTAATTAAGTGTTG GACCATCTCAGAGT-3' + pMdRbcS-REV, GGCGC GCCATGGCATTGCTCTCTCTCTCTGTTTTCTCTTC;

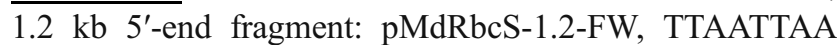
GACGGTGATAAATCATTTTTGTCT + pMdRbcS-REV; $0.5 \mathrm{~kb}$ 3'-end fragment: tMdRbcS-FW, 5'-CCATGG TACCGTTGTTCTACAATTTTCATAAATAATGTTGT-

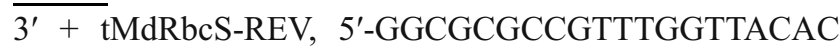
TACCCTAAGGTC- $3^{\prime}$ ). The promoter and terminator fragments included the $M d R b c S$ 3'-UTR and 5'-UTR, respectively. For cloning of the promoter sequences, $\mathrm{PacI}$ and $A s c \mathrm{I}+N c o$ I restriction sites were attached to the forward and reverse primers sequences, respectively (restriction site sequences underlined), and for cloning of the terminator, $N c o \mathrm{I}+K p n \mathrm{I}$ and $A s c \mathrm{I}$ restriction sites were attached to the forward and reverse primers, respectively. The amplified $M d R b c S$ promoter and terminator sequences were cloned in pGEM-Teasy, and the sequences were verified by sequencing. For promoter study, two lengths of 1,200 and $1,600 \mathrm{bp}$ of the $M d R b c S$ promoter ( $p 1.2 M d R b c S$ and $p 1.6 M d R b c S)$ were combined with the 
terminator $(t M d R b c S)$ sequence by introducing the terminator as NcoI-AscI fragment behind the promoter sequences. Both resulting vectors $(\mathrm{p} 1.2+\mathrm{tMdRbcS}$ and $\mathrm{p} 1.6+$ tMdRbcS) contain the $M d R b c S$ promoter and terminator sequences separated by an NcoI and a KpnI site. As reporter gene, an intron containing $\beta$-glucuronidase (GUS) gene (gus) was amplified using the vector pCambia1301 (GenBank accession number AF234297) as template. For this, a forward primer starting at the gus start codon (Gus-start, 5'-CCATGGTCCGTCCTGTA GAAACCCCAAC-3') and a reverse primer located at the gus stop codon (Gus-stop, 5'-GGTACCTCATTG TTTGCCTCCCTGCTGCGGTT-3') were used. The forward and reverse primers were extended with an NcoI and KpnI site, respectively, to facilitate cloning. As a control, the promoter of the cauliflower mosaic virus $35 \mathrm{~S}$ gene ( $p$ CaMV35S) and the terminator of the Agrobacterium tumefaciens nopaline synthase gene (tnos) were used, and these were also amplified from pCambia1301, together with the gus reporter gene. For amplification of $p C a M V 35 S+g u s$, a primer designed at $p C a M V 35 S$ (p35S-FW, 5'-TTAATTAACATGGAGTCAAAGATTCA AATAGAG-3'), which was extended with a PacI site, was used together with primer Gus-stop, and for amplification of gus + tnos, a primer designed at tnos (tnos-REV, 5'-GGCGCGCCCGATCTAGTAACATAGATGACAC-3'), which was extended with a AscI site, was used together with primer Gus-start. Finally, a fragment with $p$ CaMV35S + gus + thos was amplified using primers p35S-FW and tnos-REV. The amplified gus, pCaMV35S-gus, gus-tnos, and $p C a M V 35 S+g u s+$ thos fragments were cloned in pGEM-Teasy, and the sequences were verified by sequencing. The gus fragment was introduced as NcoI-KpnI fragment into both vectors p1.2+ tMdRbcS and p1.6+ tMdRbcS, which were opened by digestion with NcoI$K p n I$. The $p C a M V 35 S$-gus fragment was combined with the $M d R b c S$ terminator by introducing it as PacI-KpnI fragment in vector $\mathrm{p} 1.2+\mathrm{tMdRbcS}$, which was opened by digestion with $P a c \mathrm{I}$ and KpnI, thereby removing the $M d R b c S$ promoter, and the gus-tnos fragment was combined with both $M d R b c S$ promoters by introducing it as NcoI-AscI fragment in vector p1.2+ tMdRbcS or p1.6+ tMdRbcS, which were opened by digestion with $N c o$ I and $A s c$ I, thereby removing the $M d R b c S$ terminator. In total six different combinations of promoter and terminator sequences were constructed ( $p 1.2 M d R b c S$-gus-tMdRbcS; p1.2MdRbcS-gus-tnos; p1.6MdRbcS-gus-tMdRbcS; p1.6MdRbcS-gus-tnos; pCaMV35S-gus-tMdRbcS, and CaMV35S-gus-tnos (see Fig. 1)), and these were introduced as $A s c$ I-PacI fragments in the multiple cloning site of the binary vector pBINplus (van Engelen et al. 1995). The ultimate constructs were transferred to the supervirulent A. tumefaciens strain Ag10 (Lazo et al. 1991).

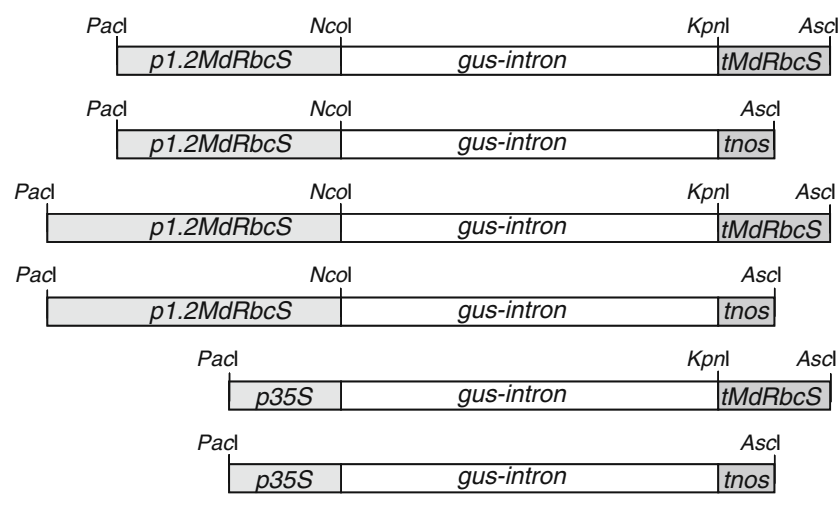

Fig. 1 Schematic representation of the different promoter-terminator combinations used for plant transformation

Plant transformation

For expression analysis of the cloned promoter fragments, transgenic tobacco (Nicotiana tabacum cv. Samsung) plants containing T-DNA with the different promoter-gus terminator constructs were produced according to Horsch et al. (1985). For each construct, 15 independent kanamycinresistant transgenic tobacco lines were selected, and per construct two to four transgenic lines showing intense GUS staining of leaf tissue were selected for further analysis.

Apple cv. Gala was transformed with the $p 1.6 \mathrm{MdRbcS}$ gus-tMdRbcS constructs only. Transformation was performed according to Puite and Schaart (1996).

Histochemical GUS assay

Histochemical GUS staining of plant material was performed as described by Jefferson (1987) using a modified staining solution containing $1 \mathrm{mM}$ 5-bromo-4-chloro-3indolyl $\beta$-glucuronide (X-gluc) in $50 \mathrm{mM}$ sodium phosphate buffer $(\mathrm{pH} 7.5), 10 \mathrm{mM}$ EDTA, $0.1 \%(v / v)$ Triton $\mathrm{X}-100,0.5 \mathrm{mM}$ potassium ferricyanide, and $5 \%(w / v)$ polyvinylpyrrolidone- 40 .

\section{Quantitative (RT)-PCR}

For quantification of gus, expression levels and of gus gene copy number quantitative (RT)-PCR was performed. For quantification of gus expression levels, total RNA was isolated from in vitro leaves of $N$. tabacum transgenic plants basically as described by Asif et al. (2000). For each transgenic line, two independent RNA isolations were performed. cDNA was synthesized using the IScript firststrand cDNA synthesis system for RT-PCR (Biorad) according to the instruction manual. For quantification of gus gene copy number, genomic DNA was isolated using the CTAB method of Doyle and Doyle (1987). Quantitative (RT)-PCR was performed as described by Schaart et al. 
(2002) using the MyiQ Single-Color Real-Time Detection System (BioRad, USA), but instead of the fluorogenic TaqMan probes, SYBR Green was used for detection of PCR products. For amplification of gus, the forward and reverse gus-primers (5'-CGGAAGCAACGCGTAAACTC$3^{\prime}$ and $5^{\prime}$-TGAGCGTCGCAGAACATTACAT-3') were used (product size $80 \mathrm{bp}$ ). As endogenous control, the elongation factor $1 \alpha$ gene $(E F 1 \alpha)$ from $N$. tabacum (GenBank accession number AF120093) was selected as a reference gene (forward $E F 1 \alpha$ primer 5'-GATTGGTGG AATTGGTACTGTC-3', reverse EFl $\alpha$ primer 5'-AG CTTCGTGGTGCATCTC-3', product size $130 \mathrm{bp}$ ). All PCR reactions were performed in duplo. For the real-time analysis, PCR products were detected directly by monitoring the increase in fluorescence from the incorporated SYBR green dye. The amplification was plotted as the normalized reporter signal, $\Delta R_{\mathrm{n}}$ (the reporter dye was normalized to the internal reference dye and corrected for the baseline value), toward the number of PCR cycles. For each reaction, the threshold cycle, $C_{\mathrm{T}}$, which is defined as the PCR cycle at which a statistically significant increase of $\Delta R_{\mathrm{n}}$ is first detected, was determined. The relative quantification was done using the comparative $C_{\mathrm{T}}$-method in which the differences in the $C_{\mathrm{T}}$ for the gus-amplicon and the $C_{\mathrm{T}}$ for the endogenous control $E F 1 \alpha$, called $\Delta C_{\mathrm{T}}$, were calculated to normalize for the differences in the total amount of cDNA or gDNA copies present in each reaction and for cDNA, the efficiency of the RT step. For comparison of two samples, the $\Delta C_{\mathrm{T}}$ values were subtracted from each other, giving a $\Delta \Delta C_{\mathrm{T}}$ value, and finally, the relative amount of gus mRNA or gDNA copies was calculated by $2^{-\Delta \Delta C_{\mathrm{T}}}$.

\section{Results}

Isolation of $M d R b c S$ promoter and terminator fragments

Using a genome walking protocol, several DNA fragments were amplified from the 5'- and 3'-flanking genomic regions of an $M d R b c S$ gene. For this reverse primers located around the start-codon of the $M d R b c S$ gene (together with adaptor primers) were designed for amplification of putative promoter sequences, and primers located around the stop-codon of the $M d R b c S$ gene were designed for amplification of a putative terminator sequence. Two putative promoter fragments of 0.9 and $1.6 \mathrm{~kb}$ were cloned and sequenced (GenBank accession numbers HM222640 and HM222639, respectively), and a single putative terminator fragment was also cloned and sequenced (GenBank accession number HM222641). The first $260 \mathrm{bp}$ of both promoter sequences near the transcription start showed a high level of similarity (91\% identity) but more distal from transcription (from start similarity was low (46\% identity)). Blasting of the promoter sequences showed that they were sharing partial homology to promoter sequences of $R b c S$ of different other plant species. Both putative $M d R b c S$ promoter sequences contain a TATA-box and several other putative cis-acting elements like two GATA-boxes (Fig. 2), which are known for their light responsive activity (Castresana et al. 1988). Also an element showing similarity to a GT-1 binding box II, which was identified in the promoter of pea $R b c S-3 A$ (Green et al. 1987), is present in the $M d R b c S$ promoter. This element is associated with high expression levels of $R b c S-3 A$ in pea. Polymorphisms that discriminate both promoter fragments were all located outside the predicted cis-acting elements (Fig. 2). The terminator sequence contained two polyadenylation signals, 70 and $116 \mathrm{bp}$ downstream of the translational stop codon.

\section{Construction of expression vectors}

For the cloning of the promoter, two sizes of 1.2 and $1.6 \mathrm{~kb}$ were taken. The promoter fragments were amplified from genomic DNA using forward primers that were based on one of the cloned 5'-flanking sequences of the $M d R b c S$ gene. The reverse primer was located directly upstream of the start-codon of the $M d R b c S$ gene, so the amplified sequence included the 5'-UTR. Both promoter sequences differed only in a few single nucleotide polymorphisms. For cloning of the $M d R b c S$ terminator, a similar approach was followed using a forward primer just downstream of the stop-codon of $M d R b c S$ (so, including the $3^{\prime}$-UTR) and a reverse primer that was based on the cloned 3'-flanking sequence of the $M d R b c S$ gene. For convenient cloning, appropriate restriction sites were included into the primers. For comparison of the regulatory function of the isolated

\section{CTGACCAAAAYAAGAGGCSAACCACCATCACACATTCACCTTCCACCTCCCTTGGATTA GATAAGATRAtgGatCTCCTTCCACGTGGCAAGGTCATAGTGGTGCMTRGTGATAAGGC TWRCWTTCAAATTGTTCCTCTGCTTCA|TGTGGTCTATATGCTGTAATGTGTCATCAKKT ACATCAYCYAACGGTCTGTGTTTGCTGGTAAAATGTTCATGGRATCAGKACCGTAGGAT AKGACAWKGSTAGTCRGAGCACTATATATA}

Fig. 2 The consensus sequence of the first $270 \mathrm{bp}$ of the MdRbcS promoter, based on both isolated fragments. The consensus contains several putative cis-acting elements. Polymorphisms are given as IUPAC ambiguity codes, and lowercase-denoted nucleotides are indel polymorphisms. Light-gray shaded and not boxed, GATA-box; darkgray shaded and not boxed, G-box; non-shaded box, Pea RbcS GT-1like box; light-gray and boxed, TATA-box 
Fig. 3 Relative gus expression levels in leaves of transgenic tobacco plants. Numbers on the $x$-axis correspond to independent transgenic lines. For each transgenic line, RNA was isolated from young leaves pooled from three different in vitro grown plants, and RNA isolation was done in duplicate. Error bars represent \pm standard error

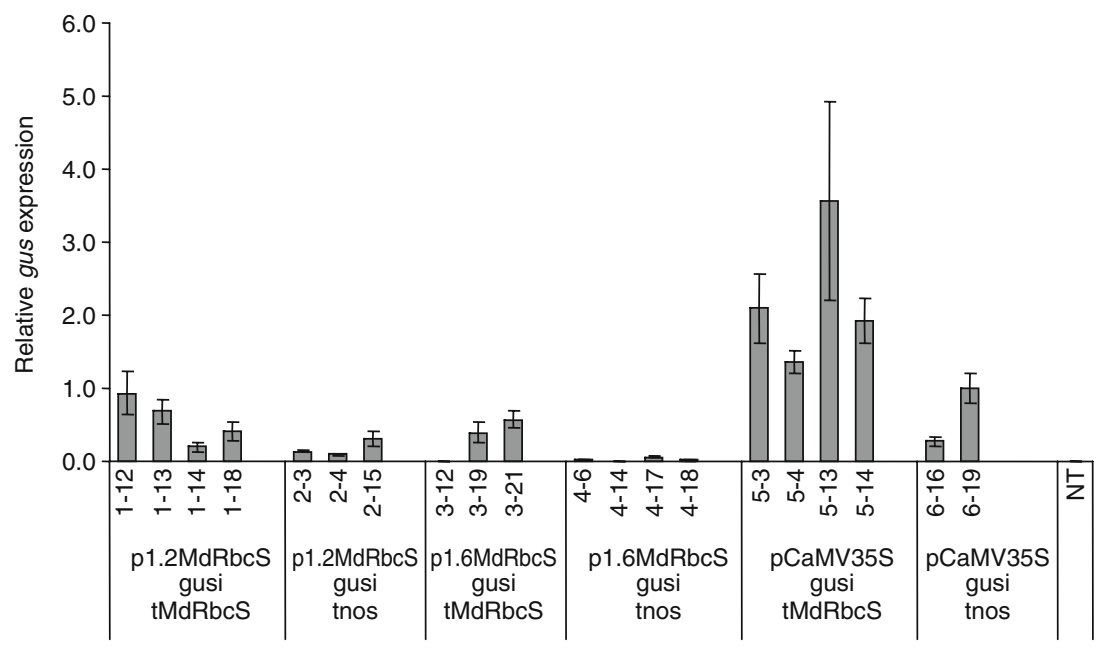

promoter and terminator sequences, the CaMV35S promoter and the nos terminator were used as reference. The six possible combinations of the three promoters $(p 1.2 \mathrm{MdRbcS}$, $p 1.6 M d R b c$, or $p C a M V 35 S)$ with the two terminators ( $t M d R b c S$ or tnos) were made and combined with the gus reporter gene and cloned into the binary vector $\mathrm{pBinplus.}$

Expression study in tobacco and apple

The different promoter-gus terminator constructs were transformed to tobacco, and for each construct, approximately 15 independent kanamycin-resistant lines were selected. In vitro leaves from all transgenic lines were checked for gus-expression by a histochemical GUS-assay. For each construct, the transgenic lines showed variation in staining intensity from low to high level of blue staining (results not shown), indicating that all promoter-terminator combinations were able to drive gus expression to high levels. No clear difference in staining intensity was found between plants transformed with the different constructs. For each construct, a number of independent transformed tobacco lines, which showed a clear uniform blue staining of in vitro leaf tissue plant, was selected for quantitative gene expression analysis. Using quantitative RT-PCR, it was determined that on average, lines with the gus reporter gene under regulation of the $M d R b c S$ promoter showed a lower gus expression level than lines transformed with pCaMV35S-gus (Fig. 3; Table 1). Both MdRbcS promoters did not differ significantly in their activity when combined with the $M d R b c S$ terminator, but when combined with the nos terminator, gus expression driven by $\mathrm{p} 1.6 \mathrm{MdRbcS}$ was significantly lower (unpaired Student's $t$ test; $P<0.05$ ) than for $\mathrm{p} 1.2 \mathrm{MdRbcS}$. For all three promoters used, the presence of the terminator sequence of $M d R b c S$ resulted in significantly higher expression levels when compared to the use of the nos terminator (Fig. 3; Table 1). The use of the $M d R b c S$ promoters combined with the $M d R b c S$ terminator gave comparable gus expression levels as the CaMV35S promoter combined with thos did.

To investigate the effect of transgene copy number on the transgene expression level for the different plant lines, the relative transgene copy number was estimated using QPCR (Table 2). The average $\Delta C_{\mathrm{T}}$ value of a group of plant lines with a corresponding large $\Delta C_{\mathrm{T}}$ value, and thus a

Table 1 Comparison of average gus expression levels in leaves of transgenic tobacco lines transformed with the different promoter-gus terminator constructs

\begin{tabular}{llllll}
\hline Plant line & Promoter & Reporter & Terminator & Average expression & tMdRbcS vs tnos \\
\hline 1 & $p 1.2 M d R b c S$ & gusi & tMdRbcS & $0.88( \pm 0.32)$ & 3.1 \\
2 & $p 1.2 M d R b c S$ & gusi & thos & $0.28( \pm 0.11)$ & 11.1 \\
3 & $p 1.6 M d R b c S$ & gusi & tMdRbcS & $0.51( \pm 0.29)$ & 3.5 \\
4 & $p 1.6 M d R b c S$ & gusi & thos & $0.05( \pm 0.02)$ & $3.5( \pm 0.90)$ \\
5 & $p C a M V 35 S$ & gusi & tMdRbcS & $1.0( \pm 0.52)$ & \\
6 & $p C a M V 35 S$ & gusi & thos & \\
\hline
\end{tabular}

For each line, the average gus expression level is calculated from the different independent transgenic events (see Fig. 3) belonging to this line. Standard deviation is given in parenthesis. For transgenic plant lines having the same promoter, but a different terminator, the relation of gus expression in lines with $t M d R b c S$ and tnos is given 
Table 2 Estimated gus transgene copy number

$\Delta \Delta C_{\mathrm{T}}$ was calculated by using the average $\Delta C_{\mathrm{T}}($ gus-EF1 $\alpha)$ of the low copy number plant lines $1-13,1-18,2-4,5-3,5-14$ as calibrator

\begin{tabular}{lllll}
\hline Plant lines & $\Delta C_{\mathrm{T}}($ gus-EF1) & $\Delta \Delta C_{\mathrm{T}}$ & $2^{-\Delta \Delta C_{\mathrm{T}}}$ & Relative copy number \\
\hline $1-12$ & 3.11 & -1.4 & 2.6 & $2-3$ \\
$1-13$ & 4.87 & 0.4 & 0.8 & 1 \\
$1-14$ & 1.91 & -2.6 & 5.9 & 6 \\
$1-18$ & 4.18 & -0.3 & 1.2 & 1 \\
$2-3$ & 2.22 & -2.2 & 4.7 & 5 \\
$2-4$ & 4.08 & -0.4 & 1.3 & 1 \\
$2-15$ & 3.15 & -1.3 & 2.5 & $2-3$ \\
$3-12$ & 2.19 & -2.3 & 4.8 & 5 \\
$3-19$ & 0.87 & -3.6 & 12.1 & 12 \\
$3-21$ & 3.11 & -1.4 & 2.6 & $2-3$ \\
$4-6$ & 1.22 & -3.2 & 9.5 & 9 \\
$4-14$ & 1.63 & -2.8 & 7.1 & 7 \\
$4-17$ & 2.81 & -1.7 & 3.1 & 3 \\
$4-18$ & 1.41 & -3.1 & 8.3 & 8 \\
$5-3$ & 4.34 & -0.1 & 1.1 & 1 \\
$5-4$ & nd & nd & nd & nd \\
$5-13$ & 1.41 & -3.1 & 8.3 & 8 \\
$5-14$ & 4.65 & 0.2 & 0.9 & 1 \\
$6-16$ & 0.61 & -3.9 & 14.4 & 14 \\
$6-19$ & 2.76 & -1.7 & 3.3 & 3 \\
\hline & & & & \\
\hline
\end{tabular}

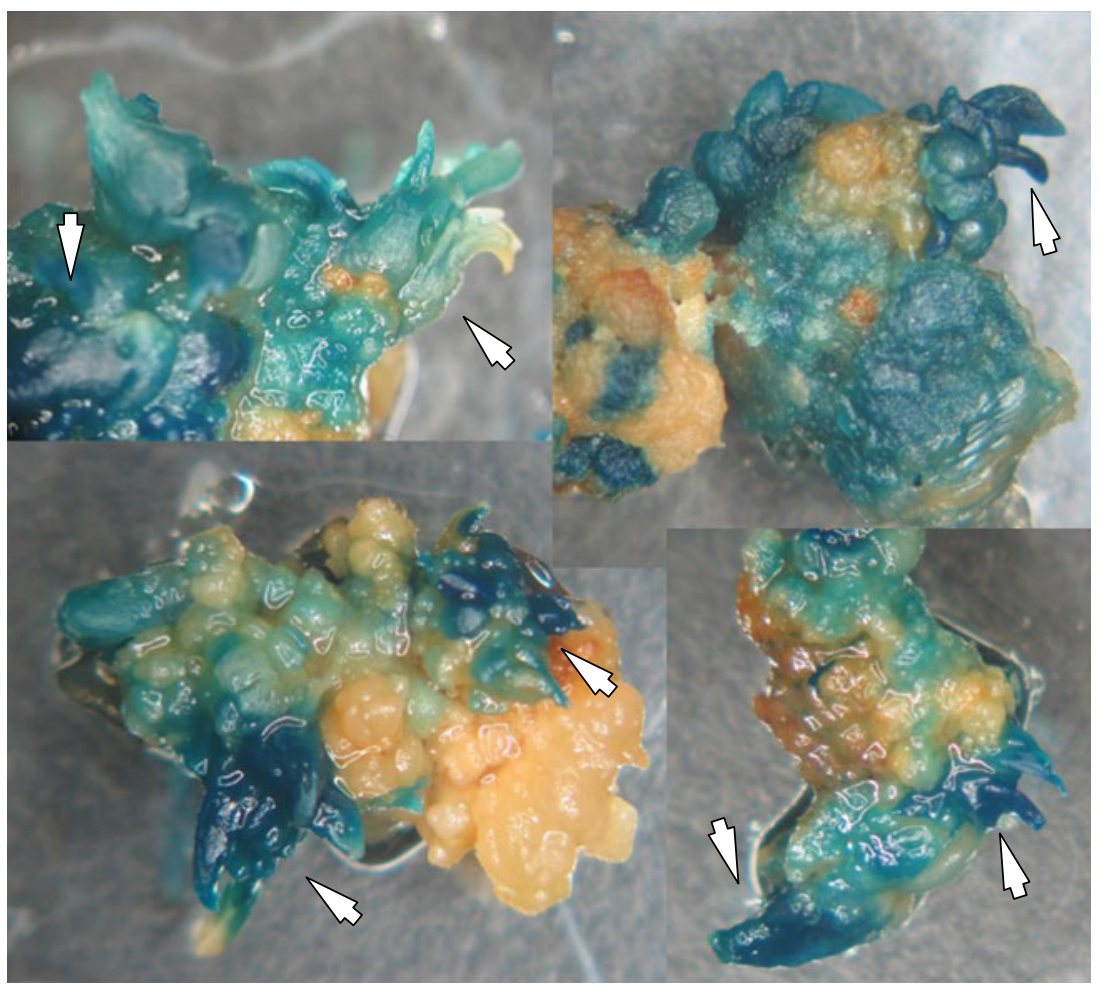

Fig. 4 Composition of four photographs showing regenerating shoots (white arrows) of apple cv. Gala that was transformed with a binary vector with $p 1.6 M d R b c S$-gus-tMdRbcS. Blue staining indicates presence of GUS activity 
similar low copy number, was used as calibrator. Half of the transgenic tobacco plants contained a relatively low number of transgene copies, but in some plant lines, high transgene copy number was found. No clear correlation was observed between transgene copy number and transgene expression level (Table 2).

In order to test the functionality of the apple rubisco promoters in transgenic apples, the p1.6MdRbcS-gus$t M d R b c S$ construct was transformed to apple cv. Gala. Several calli that produced shoots on kanamycin containing shoot regeneration medium were stained for the presence of GUS activity. Most regenerated shoots showed a strong blue staining (Fig. 4).

\section{Discussion}

For the intragenic expression of genes in apple, the availability of suitable apple regulatory sequences is desirable. Our goal was to isolate gene regulatory sequences which drive gene expression in leaf tissue to a high level comparable to that of the CaMV35S promoter. The $R b c S$ family is a photosynthesis-associated nuclear gene which is known to be highly expressed in green photosynthetic tissues and was therefore a suitable candidate to isolate regulatory sequences from. Gittins et al. (2000) tested $R b c S$ promoters of tomato and soybean in apple and concluded that they would be suitable for expression in green photosynthetic tissues. They found that $R b c S$ promoters of the different origins were able to drive gus expression to half that of plants containing the CaMV35S promoter.

Although the coding regions of different $R b c S$ gene family members are highly similar, the level and pattern of expression can vary considerably (Anisimov et al. 2007; Sawchuk et al. 2008). The apple $R b c S$ gene we used in this study to isolate regulatory sequences from shows homology to the $R b c S 1 \mathrm{~A}$ gene subfamily from Arabidopsis thaliana, which was maximally expressed in differentiating and mature leaf mesophyll cells of Arabidopsis (Sawchuk et al. 2008). Following a genome walking approach, 5'- and 3 '-flanking genomic DNA sequences of $M d R b c S$ were amplified, cloned, and characterized. In order to study the two isolated putative $M d R b c S$ promoters and the putative $M d R b c S$ terminator sequence independently, the CaMV35S promoter and the nos terminator, which are generally used in genetic modification studies, were used as reference, and different combinations of promoters and terminators with the gus reporter gene were made and tested in tobacco. Based on Q-RT-PCR analysis of transgenic tobacco leaf material, we could conclude that the $M d R b c S$ promoter was able to drive gus expression to a relatively high level, but the promoter was less active than the
CaMV35S promoter. Furthermore, Q-RT-PCR data revealed that the presence of the terminator of $M d R b c S$ had a positive effect on gus gene expression. Dean et al. (1989) reported for the first time in plants that sequences $3^{\prime}$ to the coding region (of petunia $R b c S$ ) had a significant effect on transcription rate. This finding was confirmed by Ingelbrecht et al. (1989) who showed that terminator sequences of different plant genes modulated the level of gene expression in plant cells in a different way. In their study, the presence of a terminator sequence from an Arabidopsis RbcS gene enhances transcription of the nptII gene threefold, when compared to the presence of the terminator of the A. tumefaciens octopine synthase (ocs) gene. They also showed by deletion analysis of the 3'flanking region of the ocs gene that sequences downstream of the polyadenylation signal are required for optimal gene expression. These observations indicate the importance of the terminator sequence for (trans)gene transcription. Although the $M d R b c S$ promoter combined with the nos terminator was inferior to the reference construct in which the CaMV35S promoter and the nos terminator were combined, the $M d R b c S$ promoter combined with the $M d R b c S$ terminator gave similar high gus expression as the reference construct. Therefore, the combination of both the $M d R b c S$ promoter and terminator is a suitable regulatory sequence set for the expression of transgenes to a high level in plants and for intragenesis in apple specifically. A preliminary test of the functionality of the p1.6MdRbcS-gus-tMdRbcS construct in apple resulted in an intense blue staining in a histochemical GUS staining of regeneration transgenic apple shoots, suggesting the $M d R b c S$ promoter and terminator combination to be strongly active in apple plant tissue. Whether the $M d R b c S$ promoter and terminator combination drives gene expression also to a high level in mature leaves and if the combination is also active in other tissues, for example in the fruit skin green photosynthetic active tissue, has still to be investigated. In apple, the $M d R b c S$ promoterterminator combination would be suitable to confine the expression of, for example resistance genes, to the leaves which is an important site of infection for microbial diseases and insect pests. Currently, we are testing the suitability of the $M d R b c S$ promoter and terminator for overexpression of the apple HcrVf scab resistance genes (Belfanti et al. 2004) in GM apples.

Acknowledgments This study was supported by the EU-ISAFRUIT Project (FP6-FOOD-016279). We thank Thomas Sevestre for performing the Q-(RT)-PCR experiments.

Open Access This article is distributed under the terms of the Creative Commons Attribution Noncommercial License which permits any noncommercial use, distribution, and reproduction in any medium, provided the original author(s) and source are credited. 


\section{References}

Anisimov A, Koivu K, Kanerva A, Kaijalainen S, Juntunen K, Kuvshinov V (2007) Cloning of new rubisco promoters from Brassica rapa and determination of their activity in stably transformed Brassica napus and Nicotiana tabacum plants. Mol Breed 19:241-253

Asif MH, Dhawan P, Nath P (2000) A simple procedure for the isolation of high quality RNA from ripening banana fruit. Plant Mol Bio Rep 18:109-115

Belfanti E, Silfverberg-Dilworth E, Tartarini S, Patocchi A, Barbieri M, Zhu J, Vinatzer BA, Gianfranceschi L, Gessler C, Sansavini S (2004) The HcrVf2 gene from a wild apple confers scab resistance to a transgenic cultivated variety. Proc Natl Acad Sci USA 101:886-890

Castresana C, Garcia-Luque I, Alonso E, Malik VS, Cashmore AR (1988) Both positive and negative regulatory elements mediate expression of a photoregulated CAB gene from Nicotiana plumbaginifolia. EMBO J 7:1929-1936

Dean C, Leech RM (1982) Genome expression during normal leaf development. Plant Physiol 69:904-910

Dean C, Favreau M, Bond-Nutter D, Bedbrook J, Dunsmuir P (1989) Sequences downstream of translation start regulate quantitative expression of two petunia rbcS genes. Plant Cell 1:201-208

Doyle JJ, Doyle JL (1987) A rapid DNA isolation procedure from small quantities of fresh leaf tissues. Phytochem Bull 19:11-15

Gittins JR, Pellny TK, Hiles ER, Rosa C, Biricolti S, James DJ (2000) Transgene expression driven by heterologous ribulose-1, 5bisphosphate carboxylase/oxygenase small-subunit gene promoters in the vegetative tissues of apple (Malus pumila Mill.). Planta 210:232-240

Green PJ, Kay SA, Chua N-H (1987) Sequence-specific interactions of pea nuclear factor with light-responsive elements upstream of the rbcs-3A gene. EMBO J 6:2543-2549

Horsch RB, Fry JE, Hoffman NL, Eichholtz D, Rogers SG, Fraley RT (1985) A simple and general method for transferring genes into plants. Science 227:1229-1231

Ingelbrecht LW, Herman LMF, Dekeyser RA, Van Mountagu MC, Depicker AG (1989) Different 3' end regions strongly influence the level of gene expression in plant cells. Plant Cell 1:671-680

Jefferson RA (1987) Assaying chimeric genes in plants: the GUS gene fusion system. Plant Mol Biol Rep 5:387-405

Jensen RG, Bahr JT (1977) Ribulose 1,5-bisphosphate carboxylaseoxygenase. Ann Rev Plant Physiol 28:379-400
Khoudi H, Vezina LP, Mercier J, Castonguay Y, Allard G, Laberge S (1997) An alfalfa rubisco small subunit homologue shares cisacting elements with the regulatory sequences of the RbcS-3A gene from pea. Gene 15:343-351

Lazo GR, Stein PA, Ludwig RA (1991) A DNA transformation competent Arabidopsis genomic library in Agrobacterium. Biotechnol 9:963-967

Lusk JL, Sullivan P (2002) Consumersacceptance of genetically modified foods. Food Technol 56:32-37

Nomura M, Katayama K, Nishimura A, Ishida Y, Ohta S, Komari T, Miyao-Tokutomi M, Tajima S, Matsuoka M (2000) The promoter of $\mathrm{rbcS}$ in a $\mathrm{C} 3$ plant (rice) directs organ-specific, light-dependent expression in a C4 plant (maize), but does not confer bundle sheath cell-specific expression. Plant Mol Biol 44:99-106

Outchkourov NS, Peters J, de Jong J (2003) The promoter-terminator of chrysanthemum rbcS1 directs very high expression levels in plants. Planta 216:1003-1012

Puite KJ, Schaart JG (1996) Genetic modification of the commercial apple cultivars Gala, Golden Delicious, and Elstar via an Agrobacterium tumefaciens-mediated transformation method. Plant Sci 119:125-133

Rommens CM, Humara JM, Ye J, Yan H, Richael C, Zhang L, Perry R, Swords K (2004) Crop improvement through modification of the plant's own genome. Plant Physiol 135:421-431

Sawchuk MG, Donner TJ, Head P, Scarpella E (2008) Unique and overlapping expression patterns among members of photosynthesis-associated nuclear gene families in Arabidopsis. Plant Physiol 148:1908-1924

Schaart JG, Salentijn EJM, Krens FA (2002) Tissue-specific expression of the $\beta$-glucuronidase reporter gene in transgenic strawberry (Fragaria $\times$ ananassa) plants. Plant Cell Rep $21: 313-319$

Schouten HJ, Jacobsen E (2008) Cisgenesis and intragenesis, sisters in innovative plant breeding. Trends Plant Sci 13:260-261

Schouten HJ, Krens FA, Jacobsen E (2006) Cisgenic plants are similar to traditionally bred plants. EMBO Rep 7:750-753

van der Linden CG, Wouters DCAE, Mihalka V, Kochieva EZ, Smulders MJM, Vosman B (2004) Efficient targeting of plant disease resistance loci using NBS profiling. Theor Appl Genet 109:384-393

van Engelen FA, Molthoff JW, Conner AJ, Nap J-P, Pereira A, Stiekema WJ (1995) pBINPLUS: an improved plant transformation vector based on pBIN19. Transgenic Res 4:288-290 